\title{
Communicating Mental Health Support to College Students During COVID-19: An Exploration of Website Messaging
}

\author{
Erica J. Seidel ${ }^{1}\left[\right.$ D Jan MohIman ${ }^{2} \cdot$ Corey H. Basch ${ }^{3} \cdot$ Joseph Fera $^{4} \cdot$ Alison Cosgrove $^{3} \cdot$ Danna Ethan $^{5}$
}

Published online: 7 August 2020

(c) Springer Science+Business Media, LLC, part of Springer Nature 2020

\begin{abstract}
College counseling centers are assumed to play a vital role in addressing students' mental health needs during the COVID-19 pandemic. The aim of this study was to characterize the extent to which NYC metropolitan area school websites communicated to students their updated mental health service offerings, psychoeducational information related to COVID-19, and community-based resources after the abrupt end to on-campus services during the Spring semester. A total of 138 websites were analyzed. Overall, only half of the surveyed web pages provided information about remote counseling, and just under two thirds of schools $(57.97 \%$ ) had directions for students experiencing a mental health emergency. As predicted, enrollment size was associated with whether psychoeducation about mental health and COVID-19 and information about remote counseling were available on a school's website. In both cases, medium-sized schools were the most likely to have these resources available on their websites as compared to small and large-sized schools. College counseling center web pages should include robust and current information that targets schools' diverse student bodies.
\end{abstract}

Keywords COVID-19 $\cdot$ College students $\cdot$ Mental health $\cdot$ Website messaging

\section{Introduction}

College counseling centers play a vital role in student support services. This has become even more evident during the COVID-19 pandemic; throughout the crisis, counseling centers have been on the front lines in addressing mental health issues that can derail students' academic success. The heightened severity of mental health issues among college students is well documented as is their recently increased use of campus counseling centers [1-3]. Findings from the Penn State University's Center for

Erica J. Seidel

eseidel@bmcc.cuny.edu

Jan Mohlman

mohlmanj@wpunj.edu

Corey H. Basch

baschc@wpunj.edu

Joseph Fera

JOSEPH.FERA@lehman.cuny.edu

Alison Cosgrove

Cosgrovea2@student.wpunj.edu

Danna Ethan

DANNA.ETHAN@lehman.cuny.edu
Collegiate Mental Health (CCMH) 2019 annual report on mental health trends across U.S. colleges and universities show an increase in anxiety and depression over the last 8 years among students receiving mental health treatment [4]. Students surveyed in the American College Health Association's spring 2019 National College Health Assessment reported significant levels of distress. Roughly two thirds of students reported feeling overwhelming anxiety within the last year and almost half reported they had been so depressed that it was difficult to function within the same time frame [5]. Between fall 2009 and spring 2015,

1 Department of Student Life/Counseling Center, City University of New York-Borough of Manhattan Community College, 199 Chambers Street, S-343, New York, NY 10007, USA

2 Department of Psychology, William Paterson University, Wayne, NJ, USA

3 Department of Public Health, William Paterson University, Wayne, NJ, USA

4 Department of Mathematics, City University of New YorkLehman College, Bronx, NY, USA

5 Department of Health Sciences, City University of New York-Lehman College, Bronx, NY, USA 
counseling center utilization increased by $30-40 \%$ which far outpaced the 5\% university enrollment increase during that time period [4].

The various mental health effects stemming from the COVID-19 pandemic, like the virus itself, are only beginning to be understood [6]. Data collected in April 2020 from over 2000 college students by Active Minds, a national nonprofit organization dedicated to mental health education and awareness-building for college students, showed that the pandemic had negatively impacted $80 \%$ of those surveyed [7]. The same survey indicated that $55 \%$ of college students surveyed would not know how to access mental health services from a professional when experiencing a crisis [7]. Similarly, Redden reports that whereas college students are experiencing elevated levels of stress and depression associated with COVID-19, they are at the same time finding it more difficult than usual to access sources of mental health care [8].

Other preliminary data have indicated a sharp increase in use of crisis-based services, as opposed to conventional weekly psychotherapy. The New York City metropolitan area was COVID-19's initial epicenter for morbidity and mortality in the U.S. The New York City-based crisis and support hotline, NYC WELL, saw their calls rise by about $50 \%$ in the month after the city's shutdown in response to the rapid rise in COVID-19 cases in the area [9].

College counseling centers play a critical role in addressing students' pandemic-related (and other) mental health crises. During the pandemic, the role of the typical counseling center has changed from a clinic-like setting serving face-to-face consumers, to more of a repository for web-based information, and remotely offering strategies for maintaining interpersonal connections and emotional support [6].

Accordingly, the aim of this study was to better understand the extent to which NYC metropolitan area school websites communicated to students their updated mental health service offerings, psychoeducational information related to COVID-19, and community-based resources during the Spring 2020 semester. It was hypothesized that the majority of web-based offerings from college counseling centers during the pandemic would include basic information about COVID-19, details of university services and groups that would be available during the pandemic, and other off campus self-help resource referrals. Last, because generally speaking, larger and wealthier colleges are better prepared to manage crises such as pandemic illness [10], it was expected that size in terms of enrollment would show a positive association with the most helpful aspects of counseling center websites (e.g., schools with higher enrollment would list and offer more resources to students during the pandemic).

\section{Methods}

In this cross-sectional study, all colleges and universities in the NYC metropolitan area, which encompassed areas of New York, Connecticut, and New Jersey [11] were obtained from a comprehensive list of national institutions [12]. Colleges and universities from the aforementioned geographic area with counseling centers or services available to students were evaluated. The link to the counseling centers was derived from the homepage, each page was visited, and pertinent information was categorized.

There were eight criteria evaluated in this study derived from findings from the 2018 to 2019 Healthy Minds Study [13]. The first category noted if the school had any COVID19 specific messaging on its website. This could have been presented as a full webpage, a link, an article on the website, a banner, or a video. The second category pertained to the presence of psychoeducation about mental health as it relates to COVID-19. In order for a counseling center website to be included in this category, explicit communication regarding services available during the COVID-19 pandemic must be present. Similarly, the third and fourth categories referred to the presence of information about remote counseling services offered during the pandemic and how students could access counseling services through the school. The fifth category related to access to emergency resources during COVID-19. If a school counseling center provided links to local, national, or online resources for students who were having a mental health emergency it was included in this category. The sixth category noted the presence of additional resources, such as websites, hotlines, and warmlines, while the seventh category was exclusively used for listing all resources which were found on a school's counseling website. The eighth and final category asked if all of the links provided in the seventh category were actively working. It is important to note that phone numbers and text lines were not tested. All items were coded dichotomously, with the exception of category seven which entailed listing specific resources.

In total, information from 153 college/university websites was collected for this study. From these 153 websites, 15 contained no link to a counseling center. These sites were eliminated from the analysis and, hence, 138 sites are considered in this study. Each of the 138 websites were analyzed to establish whether or not they contained predetermined counseling resources available. Independent Chi-square tests $(\alpha=0.05)$ were conducted to determine if the school's size was associated with whether or not a given counseling resource was available on the school's website. Data organization and analysis was completed using Microsoft Excel. The IRB at the City University of New York does not review studies such as this which do not include human subjects. 


\section{Results}

From the 138 schools considered, 93 (67\%) are located in New York, $14(10 \%)$ in Connecticut, and $31(23 \%)$ in New Jersey. A school was said to be small if it had $<5000$ students enrolled, medium-sized if it had 5000 to 10,000 students enrolled, and large if it had $>10,000$ students enrolled. The average enrollment across all of the schools was 6285 students with $72(52 \%)$ considered small, 40 (29\%) medium-sized, and 26 (19\%) classified as large. The cutoff points of the groups were determined by assessing the spread of the data and enabled the "medium" range to capture the median value. Then the ranges were set accordingly with this starting point.

Nearly all of the schools' websites (96\%) included COVID-19 specific messaging. Roughly $60 \%$ of all school websites included directions for students with mental health emergencies. While only $51 \%$ of all school websites studied presented information on remote counseling of any kind, $100 \%$ of those that did also provided their students with specific directions on how to access these remote services. However, only $51 \%$ of the sites included community-based remote resources, and $90 \%$ of those that did had working links.

Table 1 includes a list of predetermined counseling resources considered and provides the percentage of school websites having these resources available, broken down by a school's enrollment size. Enrollment size was significantly associated with the predetermined resources available on a school's website: psychoeducation about mental health and COVID-19 $(\mathrm{p}=0.03)$ and information about remote counseling $(p=0.03)$. Results indicated that medium-sized schools were more likely than small or large universities to provide psychoeducation about mental health and COVID-19. Medium and large-sized sized universities were more likely than small sized universities to provide information about remote counseling.

\section{Discussion}

This study explored the extent to which information about up-to-date school- and community-based mental health services and relevant psychoeducational messaging were available on counseling center webpages of NYC metropolitan area college and university websites during the first critical weeks of city and college closures due to the COVID-19 pandemic.

Perhaps due to the many unknown aspects of the pandemic as it continues to unfold, well under half of the schools' web pages included information about mental health as it specifically relates to COVID-19 (42.75\%). This will become an important aspect of the delivery of mental health services moving forward, as the unique effects of the pandemic on the emotional health of the college population are better understood [14]. Additionally, this aspect of psychoeducation should be updated regularly and kept current with scientific findings as well as city, state and national policies and recommendations.

Overall, only half of the web pages analyzed gave information about remote counseling, and just under two thirds of schools had directions for students experiencing a mental health emergency. Making this information accessible to students online is essential, especially in the absence of a physical office where those in need or who have concerns about others can seek immediate help [7]. Half of the websites surveyed had links to community-based resources. Community-based resources are particularly helpful to provide services such as round-the-clock hotlines and warmlines, particularly during hours when school counseling services are unavailable.

Enrollment size of the school was associated with whether its counseling center web page provided information about remote counseling, with medium and large-sized schools doing better than small schools. This could be due to the location of many mid- and large-sized schools in urban areas benefitting from a range of options for obtaining mental health treatment. Medium-sized schools were also most

Table 1 Percentage of 138 schools with predetermined counseling resources available on their website broken down by student enrollment size

\begin{tabular}{|c|c|c|c|c|}
\hline Resource & $\begin{array}{l}\text { Small schools } \\
<5000 \text { students } \\
\text { enrolled }(\%)\end{array}$ & $\begin{array}{l}\text { Medium schools } \\
5000 \text { to } 10,000 \mathrm{stu}- \\
\text { dents enrolled }(\%)\end{array}$ & $\begin{array}{l}\text { Large schools } \\
>10,000 \text { students } \\
\text { enrolled }(\%)\end{array}$ & All sizes $(\%)$ \\
\hline COVID-19 messaging & 93.06 & 100.00 & 96.15 & 95.65 \\
\hline Psychoeducation about Mental health and COVID-19* & 34.72 & 60.00 & 38.46 & 42.75 \\
\hline Information about remote counseling* & 40.28 & 65.00 & 57.69 & 50.72 \\
\hline Directions for students with mental health emergencies & 48.61 & 70.00 & 65.38 & 57.97 \\
\hline Links to community based remote resources & 50.00 & 55.00 & 50.00 & 51.45 \\
\hline
\end{tabular}

An $*$ indicates that enrollment size had a significant effect $(\mathrm{p}<0.05)$ on the presence of this counseling resource 
likely to have psychoeducation about COVID-19 and mental health services listed on their websites. This could be due to the balance of a tangible need for services coupled with a sense of responsibility to the student body. Future research is needed to clarify the relation of enrollment size to the type and amount of psychoeducational and treatment referral data provided on counseling center websites.

During the initial shift to remote learning, provision of continued access to quality mental health support for students required quick action from college counseling staff. With a growing recognition that the instability of this pandemic will be present for some time, it is clear that colleges and universities must adapt their understanding ofand develop innovative strategies to address-the mental health needs of their students [8]. Institutional support and prioritization of these measures will be critical for success as will be the prioritization of equity and student access to quality mental health care where students serve as active stakeholders. The results of this pilot study can be useful for counseling centers in considering how mental health messaging, services, and community connections can be improved to ensure that students have the mental health support they need to thrive in this new landscape. Issues of access to care have become even more evident and should be at the forefront of student health and wellness-related prevention, intervention strategies and delivery of services moving forward.

Author Contributions CHB, EJS, JM, and DE developed the methods for this study. EJS and DE created content categories, and AC analyzed websites based on these categories. JF analyzed and interpreted the data. EJS developed the initial draft of the manuscript and acted as corresponding author. All authors revised and contributed to the final draft of the manuscript.

Funding No funding support was received for this study.

\section{Compliance with Ethical Standards}

Conflict of interest The authors declare that they have no conflict of interest.

\section{References}

1. Canady, V. (2018). National survey reveals college-student mental health diagnosis increase. Mental Health Weekly, 28(43), 1-7. https://doi.org/10.1002/mhw.31663.
2. Levin, A. (2020). University Mental Health Centers Strain to Keep Up With Increased Demand. Psychiatric News, 55(1), 12-13. Retrieved from https://psychnews.psychiatryonline.org/ doi/full/10.1176/appi.pn.2019.12b24\#.XijovQdaB9w.email.

3. Xiao, H., Carney, D.M., Youn, S.J, et al. (2017). Are we in crisis? National mental health and treatment trends in college counseling centers. Psychological Services, 14(4), 407-415. https://doi.apa. org/doiLanding?doi=10.1037\%2Fser0000130.

4. Center for Collegiate Mental Health. (2020). 2019 Annual Report (Publication No. STA 20-244). Retrieved from https://ccmh.psu. edu/files/2020/03/2019-CCMH-Annual-Report_3.17.20.pdf.

5. American College Health Association. (2019). American College Health Association-National College Health Assessment II: Reference Group Executive Summary Spring 2019. Silver Spring, MD: American College Health Association. Retrieved from https ://www.acha.org/documents/ncha/NCHA-II_SPRING_2019_US_ REFERENCE_GROUP_EXECUTIVE_SUMMARY.pdf.

6. Anderson, G. (2020). Coping With a Pandemic. Inside Higher Ed. Retrieved from https://www.insidehighered.com/ news/2020/03/31/mental-health-support-systems-coping-pande mic.

7. Active Minds. (2020). The Impact of COVID-19 on Student Mental Health. Retrieved from https://www.activeminds.org/wp-conte nt/uploads/2020/04/Student-Survey-Infographic.pdf.

8. Redden, E. (2020). Pandemic Hurts Student Mental Health. Inside Higher Ed. Retrieved from https://www.insidehighered. com/news/2020/07/13/survey-finds-higher-prevalence-depression -among-students-and-difficulties-accessing.

9. Kunzmann, K. (2020). Mental Health Crisis Hotline Use Spikes During COVID-19. HCP Live. Retrieved from https://www. mdmag.com/conference-coverage/apa-2020/mental-health-crisi s-hotline-use-spikes-during-covid19.

10. Murakami, K. (2020). Haves and Have-Nots on COVID-19 Protection. Inside Higher Ed. Retrieved from https://www.insidehigh ered.com/news/2020/06/25/wealthier-colleges-can-offer-moreprotection-covid-19-cash-strapped-peers.

11. New York City Planning (n.d.). New York City: A city of neighborhoods. Retrieved June 30, 2020 from: https://www1.nyc.gov/ site/planning/data-maps/city-neighborhoods.page.

12. Peterson's Guide. (n.d.). Retrieved June 30, 2020 from: Peterson's Guide. https://www.petersons.com/.

13. Healthy Minds Network (2019). The Healthy Minds Study. Ann Arbor, MI: University of Michigan. Retrieved from https://healt hymindsnetwork.org/wp-content/uploads/2019/09/HMS_national2018-19.pdf.

14. June, A.W. (2020, July 13). College Students Have Been Stressed Out During the Pandemic. Here's How It's Affected Their Mental Health. The Chronicle of Higher Education. Retrieved from https ://www.chronicle.com/article/College-Students-Have-Been/24916 7 ? key $=001$ RV fHGcm vIBQW 9 w WAe oLmEQ tJg UT bof k8-cCTpBy2sJHaLi3IqXzAAIfIRtKXQ1FaVlg4YzVDeDVSUG hMNEJUR3d5UWM0Qm9IeTBLNHEzTldtZV9jbzRHYw.

Publisher's Note Springer Nature remains neutral with regard to jurisdictional claims in published maps and institutional affiliations. 